\title{
Bimodal DC-DC Converter With an Efficient Pass-Through Zone
}

\author{
Charles E. Mullett \\ ON Semiconductor \\ 732 Montclair Drive \\ Santa Paula, CA 93060 \\ Chuck.Mullett@onsemi.com
}

\begin{abstract}
A new, efficient dc-dc converter is formed by combining buck and boost stages and controlling the switches to provide a pass-through zone such that when the value of the input is close to the output voltage no switched-mode processing occurs. Efficiency is improved throughout the entire range of operation. It is ideally suited as a preregulator in automotive applications.
\end{abstract}

\section{INTRODUCTION}

The most popular nonisolated, noninverting dc-dc converter topologies either buck (lower the voltage) or boost (raise the voltage) but are not capable of handling inputs that swing both above and below the desired output. Three other topologies are capable of producing noninverted outputs less or greater than the input voltage. These are the single-ended primary inductance converter (SEPIC), the buck + boost, and the boost + buck. The latter two are simple combinations of the buck and boost, in which the two sets of switches are driven simultaneously, causing the converter to buck and boost simultaneously to form the output. It will be shown that all three of these have disadvantages, and that these disadvantages are overcome in the new bimodal converter.

\section{EXISTING TECHNOLOGY}

\section{A. Background Example---the SEPIC}

The SEPIC topology is shown in Figure 1. This circuit produces an output of the same polarity as the input, and the input voltage can be above or below the desired output.

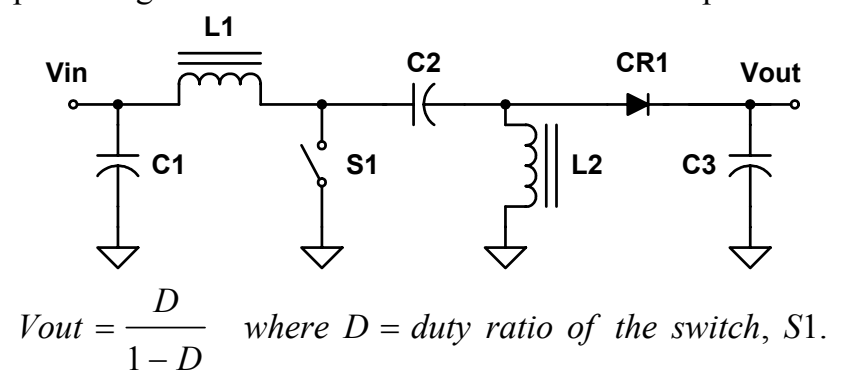

Figure 1. SEPIC.

Although the classic SEPIC contains two inductors, they may be integrated onto one core (with unity turns ratio), since they have the same voltage waveform across them.
Transposing the equation for Vout to get an expression for D

yields the equation $\quad D=\frac{\text { Vout }}{\text { Vin }+ \text { Vout }}$

It can be seen by inspection that with Vin $=$ Vout, $\mathrm{D}=0.5$. A bit more insight or analysis will show (again with Vin $=$ Vout) that when the switch is on, it conducts twice the input current, and when the diode is conducting, it also conducts twice the output current (the input and output currents are equal). It appears that considerable energy is "churned" in the converter, when its only purpose is to pass the input to the output! A final observation about the SEPIC is that the input current is smooth (due to the input inductor), the output current is chopped (due to the diode feeding the output), and all energy is passed through a capacitor.

\section{B. Background Example---Buck + Boost}

The buck + boost circuit is shown in Figure 2. In the most popular implementation of this circuit, the buck switch and the boost switch are driven together.

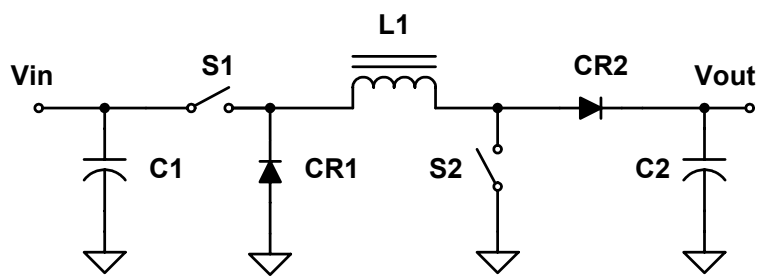

Vout $=\frac{D}{1-D}$ where $D=$ duty ratio of the switches $S 1$ and $S 2$.

Figure 2. Buck + boost converter. The two switches are driven simultaneously.

With both switches driven simultaneously at duty ratio $\mathrm{D}$, the transfer function is the same as that of the SEPIC, so

$$
D=\frac{\text { Vout }}{\text { Vin }+ \text { Vout }}
$$

In this case, both switches and both diodes conduct twice the input/output current while they are on $(50 \%$ of the period 
when Vin = Vout), and the inductor conducts twice the current continuously. This circuit "churns" even more energy than the SEPIC. Not only that, but when the input equals the output, the current in the input and output capacitors is a symmetrical square wave, having the maximum rms value and stress on the capacitors. This characteristic of chopped input and output currents is shared by the classic buck/boost and flyback topologies.

\section{THE BIMOdAl CONVERTER}

The bimodal converter uses the same power train as the buck + boost converter, but behaves in an entirely different (and much more efficient) way. As implied by the name, it operates in two different modes, and never in a combination of the two. Since Figure 2 shows only the power train, it is a valid reference for the explanation of the bimodal converter's operation. When the input is above the desired output, the buck control switch, S1, is pulse-width modulated while the boost control switch, S2, is always off. When the input is below the desired output, S1 is held on continuously (not switching) while S2 is pulse-width modulated to raise the voltage to the desired output value. When the input equals the output (neglecting the voltage drops in S1 and D2), there is no switching at all, and the power is simply handed from the input to the output with no switched-mode losses. [1] Although the circuit is the same as in Figure 2, the transfer functions are totally different:

$$
\begin{gathered}
\text { Vin }<\text { Vout }: \frac{\text { Vout }}{\text { Vin }}=\frac{1}{1-D 2} \text {, and } D 1=1 \\
\text { Vin }>\text { Vout }: \frac{\text { Vout }}{\text { Vin }}=D 1, \text { and } D 2=0
\end{gathered}
$$

where D1 and D2 are the duty ratios of switches S1and S2. As indicated by the above equations, when Vin is below Vout, the transfer function is that of the classic boost converter, and the buck switch, S1, is on continuously (D1 = 1). When Vin is above Vout, the transfer function is that of the classic buck converter. The buck and boost modes are mutually exclusive (hence, bimodal).

\section{A. Adding the Pass-Through Zone}

In many of the industrial applications of these dc-dc converters, the output drives motors, relays, actuators, etc., and the output voltage is not critical. This may even apply to intermediate bus converters in distributed power systems. In these cases the concept of passing the input to the output can be extended to a range of input voltage, or "pass-through zone" over which there is no switching, with two significant advantages: 1) There is no switching loss at all in the passthrough zone, and 2) There is less loss in the range of input voltage above and below the pass-through zone, because the converter does less work. With the input above the passthrough zone, the buck stage simply bucks the voltage down to the upper boundary of the pass-through zone. Conversely, with the input below the pass-through zone, the boost stage boosts the voltage up to the lower boundary of the passthrough zone, not up to the center value of the desired output voltage. The transfer characteristic of this new bimodal converter is shown in Figure 3.

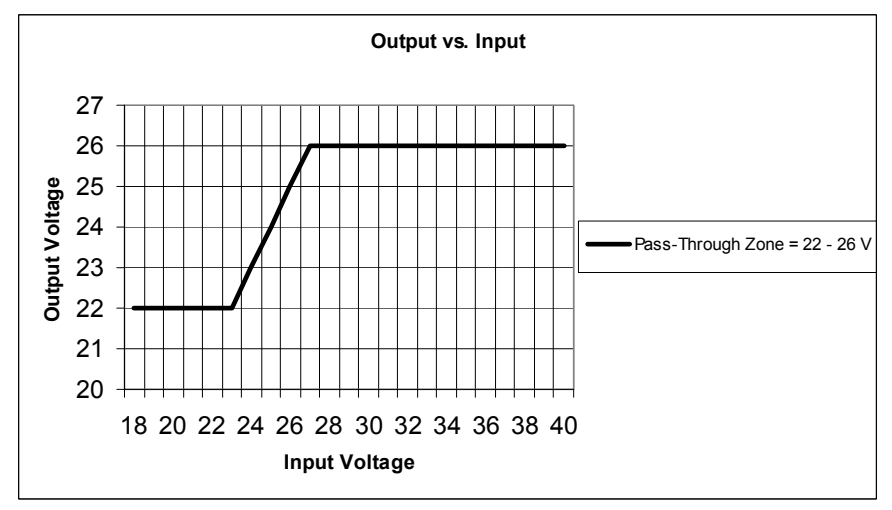

Figure 3. Transfer characteristic of the bimodal converter with passthrough zone.

\section{B. Experimental Results}

A prototype was built for the $24-\mathrm{V}$ application to demonstrate the effect of the pass-through zone, and the results are shown in Figure 4.

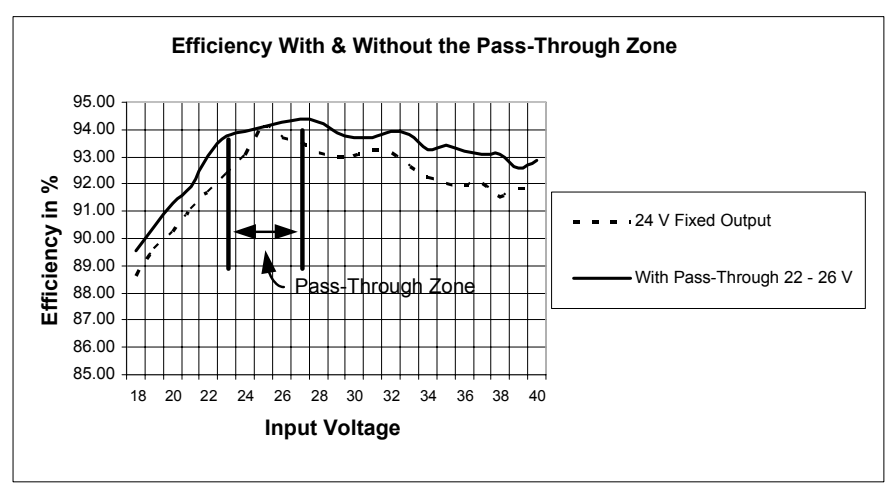

Figure 4. Efficiency of the prototype converter, showing the effect of the pass-through zone.

Note the improved efficiency, not only in the pass-through zone, but also outside it, where there is less work to do in the buck or boost process. Also note the efficiency where the input equals the output---this is the condition where, even without the intentional pass-through zone, the bimodal converter approaches a non-switching condition.

This prototype was built (from discrete parts) for simplicity, not for optimum efficiency. The switch in the buck stage was a P-channel FET, and the shunt element was a Schottky diode, not a synchronous FET. Similarly, in the boost stage, the switch was an N-channel FET, and the series element was a Schottky diode, not a FET. This saved considerable drive circuitry that would be fairly trivial in an integrated controller. With FETs replacing the diodes, the 
efficiency improvement due to the pass-through zone would be much more dramatic, because the conduction losses would be further reduced, making the switching losses (and the absence of them) much more dramatic. Also, at $12 \mathrm{~V}$ (for automotive and many industrial applications) the advantages of the bimodal topology and the pass-through zone will be even more pronounced.

\section{Control Scheme}

A simplified block diagram of converter is shown in Figure 5. This one was designed for a $24 \mathrm{~V}$ application, where the input came from an off-line transformer and full-wave rectifier with single capacitor filter, and the output was used for motors, actuators and relays in a vending machine. It would be useful also in intermediate bus converters in distributed power systems, where the output feeds point-ofload (POL) dc-dc converters. For automotive applications, it can be designed for an input range of $4-60 \mathrm{Vdc}$, with an output of 7 to $8 \mathrm{~V}$ (to feed linear $5 \mathrm{~V}$ regulators) or $5 \mathrm{~V}$ fixed (without the pass-through band). Reducing the pass band to zero is accomplished by simply replacing resistor $\mathrm{Rb}$ in the feedback divider with a jumper and readjusting the remaining resistance ratio $(\mathrm{Ra} / \mathrm{Rc})$ to achieve the desired output voltage.

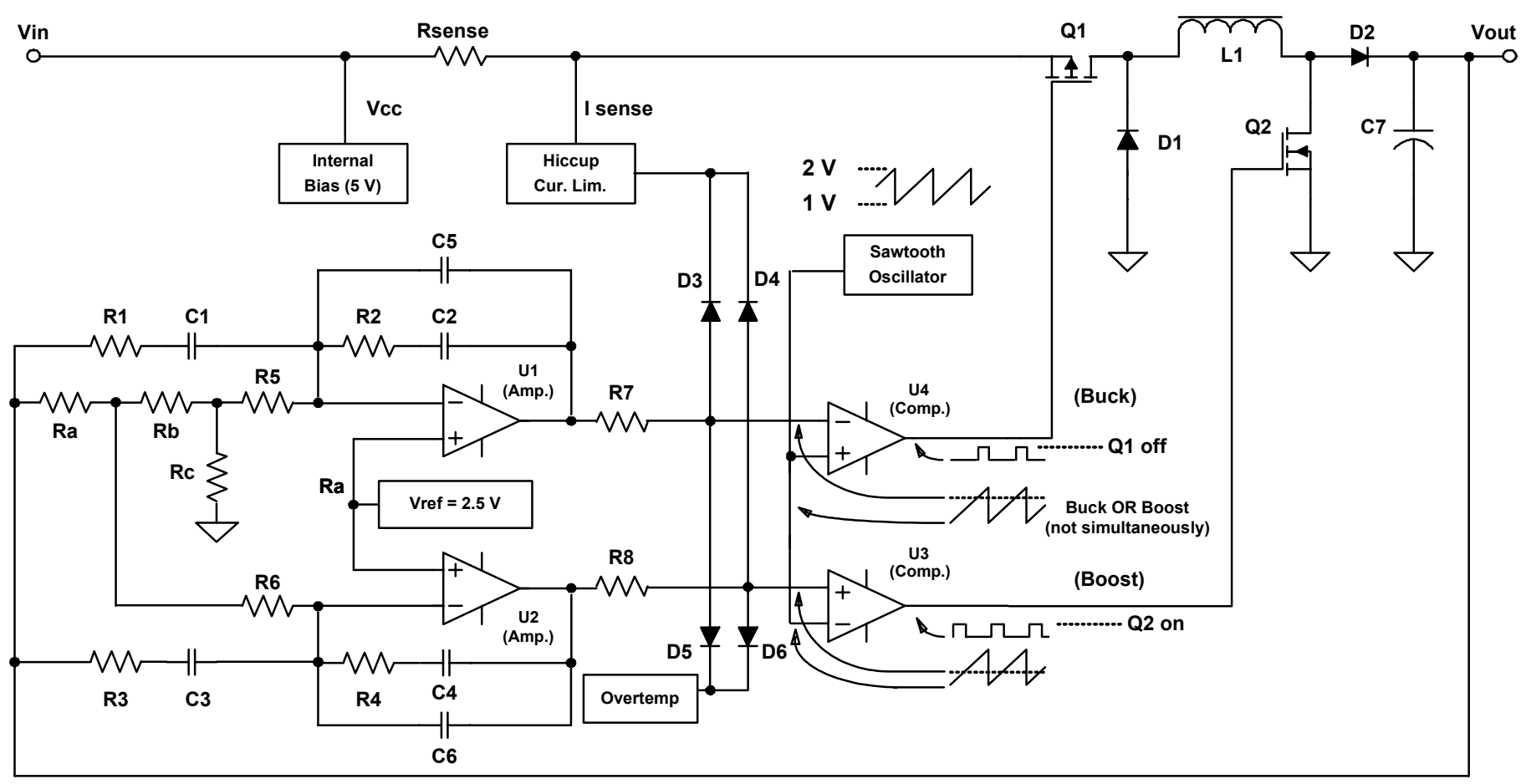

Figure 5. Simplified schematic of the bimodal converter with the pass-through zone.

\section{Detailed Operation}

The power train consists of a buck regulator, cascaded with the boost regulator and sharing a common inductor, L1. The buck section contains switch Q1, free-wheeling diode D1, and the inductor L1. The boost section contains the input inductor (again, L1), the boost switch Q2, boost diode D2, and the output capacitor, C5. The explanation that follows assumes ideal switches and diodes for simplicity. In the buck mode,

When the input voltage is above the pass band, Q1 is pulse-width modulated in the normal manner of a buck converter, while the boost switch, Q2, is always off. In this case the voltage at the top of Rc equals the reference voltage, Vref $(2.5 \mathrm{~V})$. The output of the error amplifier U1 is fed to comparator U4 for comparison to the ramp signal from the sawtooth oscillator. During the time when the ramp voltage exceeds the voltage from U1, the comparator output will be high and the buck switch, Q1, will be off. During the remaining portion of the period it will be on. During the entire period the output of amplifier U2 will be saturated in the low state, because its inverting input is above the noninverting input (Vref). Comparator U3 does not switch, because its other input (the ramp signal) is always above the saturated low level of the amplifier U2.

When the input is below the pass band, Q2 is pulse width modulated in the normal manner of a boost converter, while the buck switch, Q1, is always on. Q1 stays on, because the output of amplifier U1 is saturated at its upper limit, above the peak of the ramp waveform. This keeps the output of comparator U4 in its low state, holding the buck switch, Q1, on continuously. However, the voltage at the top of $\mathrm{Rb}$ will be equal to Vref, and the output of amplifier U1will be at a voltage within the range of the ramp signal, causing comparator U4 to pulse-width modulate the boost switch, Q2, to regulate the output at the lower boundary of the passthrough band. 
When the input voltage is within the pass-through band, amplifiers U1 and U2 are saturated high and low, respectively. This results in the buck switch, Q1, being on continuously and the boost switch, Q2, being off continuously. As a result, the input is conveyed directly to the output without any switchedmode operation at all. The efficiency is determined by the $\mathrm{R}_{\mathrm{DS} \text { (on) }}$ of $\mathrm{Q} 1$, the resistance of the current-sampling resistor, $\mathrm{R} 1$, the resistance of inductor $\mathrm{L} 1$, and the forward voltage drop of the boost diode, D2.

\section{E. Loop Compensation}

For optimum transient response in both the buck and boost modes, separate amplifier feedback networks are shown. Each is a classic "type 3" compensator. their inputs are separated by resistors R5 and R6, intended to be higher by a factor of five or ten than the resistance of the feedback voltage divider at the respective taps. If optimum transient response is not important, then the networks can be simplified by elimination of R5 and R6, and also R1, C1, and C6.

\section{F. Current Limiting}

Current limiting, covering all three modes of operation (buck, boost, and pass-band), is accomplished by sensing the voltage across a current-sensing resistor at the input. When the limit threshold is exceeded a "hiccup" type of current limiter is engaged, shutting both drives off via applying a low voltage (near ground) to diodes D3 through D6. This forces comparator U4's output high and comparator U3's output low, resulting in both Q1 and Q2 being held in their nonconducting state.

\section{F. Overtemperature Protection}

Overtemperature protection of the switches and/or the control circuitry can be sensed and the output shut off by the same technique as used for the current limiter. In both cases, the protection circuits can be either self-recovering or latching, as desired.

\section{Boost + Buck Bimodal CONVERTER}

A somewhat obvious variation of the bimodal converter described in the preceding text is formed by interchanging the buck and boost stages, forming a "boost + buck" type, as contrasted to the "buck + boost" type. This is shown below in Figure 6.

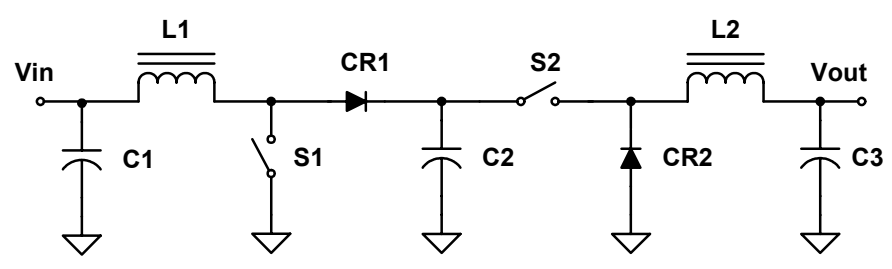

Figure 6 . Boost + buck bimodal converter.
This circuit performs with the same transfer functions as the buck + boost type previously described, and can be driven from the same control circuitry. Notice that it contains two inductors, with a capacitor in the center. This one has not been built and tested as of this writing, but analysis shows it to be a promising alternative. Because of the placement of the inductors, it has smooth input current as well as smooth output current. These are both desirable, especially at higher currents and when conducted EMI must be minimized. The expense and space penalty may well be offset by savings in filter components. Unlike the SEPIC, the inductors do not share the same waveform, so the two inductors cannot be combined. It may be possible to eliminate the center capacitor, or at least make it small and necessary only to eliminate parasitic ringing. Depending on the quality of the input and output capacitors and the losses of the added inductor, this configuration may or not be more efficient than the buck + boost approach.

\section{Automotive ApplicAtions}

The bimodal converter is particularly well-suited for applications where the input contains abnormal voltage swings, such as the "cold cranking" situation in cars and trucks. In cases where a well-regulated $+12 \mathrm{~V}$ or $+5 \mathrm{~V}$ bus is required in the presence of a battery voltage that is normally $+13.8 \mathrm{~V}$, but dips to as low as $4 \mathrm{~V}$ during starting the engine in the presence of extreme low temperatures or nonideal battery condition, the bimodal converter is ideal. It has two advantages over the SEPIC, as it does not rely on a capacitor for transfer of energy and requires only one single-winding inductor. If the output does not need to be tightly regulated, the pass-through band can enhance the efficiency. If tight output regulation is required, then the pass-through band is simply set to zero by eliminating the center resistor of the feedback voltage divider.

\section{REFERENCES}

[1] Ray Ridley, Siegfried Kern, Berthold Fuld, "Analysis and Design of a Wide Input Range Power Factor Correction Circuit for Three-Phase Applications," Proceedings of APEC 1993, pp. $299-305$.

[2] Charles E. Mullett, "DC-DC Converter for Driving HighIntensity Light-Emitting Diodes with the SEPIC Circuit," Application Note AND8138/D, available from ON Semiconductor (www. onsemi.com).

[3] "A Unique Converter Configuration Provides Step Up/Down Functions," Application Note AN954, available from ON Semiconductor (www. onsemi.com).

[4] Charles E. Mullett, "Efficient Nonisolated Up/Down DCDC Converters," Proceedings of PCIM Europe, 2004. 